\title{
Some Missing Data Patterns for Mutidimensional Scaling
}

\author{
Paul Thompson \\ University of North Camolimaิ
}

The conditional rank order (CRO) method has been suggested to reduce the information load for obtaining similarity data (Green \& Rao, 1972; Torgerson, 1958; Young, 1975). In this method one item in the set is used as a "target," and the remaining stimuli are ranked in the order of their similarity to the target. Usually, all items, one after another, are used as targets. More judgments must be made in the CRO task than in the paired comparisons task, but the judgments are easier (Young, 1975) because the previous judgments restrict the set of choices.

If there are a large number of items to scale, information overload eventually takes place in the CRO task. Many categories must be used in order to perform the task (assuming that each rank is considered a separate category). Therefore, it must be asked if even this conceptually easier task would tax the capabilities of the normal subject.

Little attention has been paid to limitations of subjects to perform similarity scaling tasks. Rather, attention has been devoted to designing procedures which collect information in fractional patterns; subjects are presented carefully designed subsets of the data (Graef \& Spence, 1979; Spence \& Domoney, 1974). These discussions suggest selecting subsets of items with large inter-item distances, and they evaluate the impact of this suggestion with monte carlo techniques. Certain assumptions are involved in these techniques. For instance, it is assumed that the same process is employed in the comparison of both similar and dissimilar stimuli. 
If this assumption is not valid, their suggestions must be reconsidered.

An attempt was made to examine limitations in the process of making $\mathrm{CRO}$ judgments. One focus of the investigation lay in assessing the quality of the configuration if the subject was allowed to make a subset of the judgments of his/her own choosing. The second focus lay in assessing whether data gathered using the CRO task resulted in unreliable data and, if so, what portion of the data were unreliable.

\section{Method}

Subjects. Forty-three students were used in this experiment. Students participated in partial fulfillment of course requirements. They were divided into three groups, two with 15 students and one with 13 students. After responses were examined, 10 students were eliminated.

Stimuli. Three sets of 15 nouns each were used as experimental stimuli. The first set was composed of animal nouns selected to be a biased subclass of the category of Common Mammals. They were selected from the animals falling in the small/domesticated animal portion of the solution obtained by Henley (1969). This will be called the Selected Animals (SA) set. This set was scaled by 13 subjects (later reduced to 10). The second set of nouns was taken from the same experiment but were chosen at random from all animal names used by Henley. This will be called the Random Animals (RA) set. There were 15 subjects in the group used to scale this set of stimuli (later reduced to 13). The third set of nouns was selected randomly from the American Heritage Dictionary. Words with two functions, such as "noun" and "verb," were excluded. These nouns had approximately the same mean length and number of syllables as the animal terms. There were 15 students in the group used to scale this set of stimuli (later reduced to 10). This will be called the Random Nouns (RN) set.

Procedure. Subjects were run in two sessions. They gave similarity judgments using the CRO method. In the first session, each subject provided 15 sets of judgments; each item served as a target, with the remaining 14 stimuli ranked in order of similarity to this target for a total of 210 judgments. No instructions as to order of decision were given (most similar first, least similar first, and so forth). No instructions about or definitions of "similarity" were given. After completing this ranking task, subjects were asked to indicate a point at which they had less confidence in their judgment, if such a point existed.

The students returned in two weeks for the second session. During this session of the experiment, 10 of the 15 stimuli were used as the targets for a given subject. These 10 stimuli were randomly chosen, such that each stimulus served as a target equally often. All 14 stimuli were ordered in relation to each target, for a total of 140 judgments. After completing the ranking task in the second session, the subjects were again asked to indicate the point in the rank order at which they had less confidence in their ranking behavior, if such a point existed.

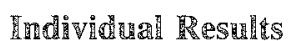

The responses of each subject during the two sessions were examined for consistency and reliability. Certain subjects were much less reliable and were eliminated from further consideration. Three methods of contrasting such subjects to the more reliable subjects were used. Correlation and mean absolute difference (MAD) values were calculated between the two sessions. The 33 retained subjects were more consistent (mean $r=.521$, mean $M A D=2.98)$ than the 10 eliminated subjects (mean $r=.203$, mean MAD $=4.05$ ). Mean profiles were computed for each target stimulus (excluding the individual's scores) and subject-group correlations were computed. The mean correlations were quite different (mean $r$ for retained subjects $=.618$; mean $r$ for eliminated subjects $=.318$ ). Clearly, those eliminated differed from the other subjects. Those eliminated found it difficult to perform the task in the second session and used inappropriate strategies (such as alphabetizing their responses). All results reported here are based on the 33 retained subjects. 


\section{Ra斯 Valte Eval}

The assignment of ranks between the two sessions was next examined. Conditional proportions are presented in Table 1 . The proportion of times that exactly the same value was assigned in both sessions is listed in the column labeled "\% Same." The proportion of consistent responses is listed under "\% Cons," which involves assigning a rank no more than one number different in Session 2. Clearly, the subjects were not consistent. The proportion of consistent rankings was above .5 only for extreme rank values, Ranks 1 through 4 and 13 through 14. The mean rank assigned in Session 2 (conditional upon the value assigned in Session 1) is also listed in Table 1 . Overall, these means increase monotonically, indicating that the subjects were performing the same task in both sessions.

Kendall's tau correlation coefficients were computed berween the rankings given to the stimuli in the different sessions $\left(r_{\mathrm{SA}}=.61, r_{\mathrm{RA}}=.59\right.$, $\left.r_{\mathrm{RN}}=.42\right)$. There seems to be little difference in the consistency between the SA and RA sets, but the RN set was much lower, probably related to familiarity difference between the sets.

\section{Multidimensional Scaling Solutions: 䠌esults from the Complete Orders}

The data were analyzed by ALSCAL (Takane, Young, \& deLeeuw, 1977). Two-dimensional solutions were obtained and compared for two reasons. First, such solutions were used because they were more economical. Secondly, the intent was to compare similar solutions, rather than to obtain an optimal solution. For this purpose, two-dimensional solutions are as good as any other.

The configurations computed from the complete orders are presented in Figures 1, 2, and 3. The data were analyzed as row-conditional, asymmetric data, under the individual differences model. Ties which arose naturally from the subjects remained tied, the secondary approach to ties (Kruskal, 1964). Only solutions obtained in Session 1 are presented, as the two sessions produced similar solutions. The solution from the complete ordering of the SA set in Session 1 is presented in Figure 1 (stress $^{1}$ for Session $1=.169$, stress for Session $2=.150$ ).

'Stress values are stress form 1 (Kruskal, 1964).

Table 1

Rank order value use in Session 2 conditional upon use in Session 1

\begin{tabular}{|c|c|c|c|c|c|c|c|c|c|}
\hline \multicolumn{4}{|c|}{ Selected Animals } & \multicolumn{3}{|c|}{ Random Animals } & \multicolumn{3}{|c|}{ Random Nouns } \\
\hline Rank & $\%$ & $\%$ & Mean & $\%$ & $\%$ & Mean & $\%$ & \% & Mean \\
\hline Order & Same & Cons & Rank & Same & Cons & Rank & Same & Cons & Rank \\
\hline 1 & .77 & .85 & 1.68 & .60 & .78 & 2.47 & .53 & .72 & 2.25 \\
\hline 2 & .41 & .66 & 3.70 & .35 & .62 & 3.60 & .30 & .68 & 3.86 \\
\hline 3 & .32 & .55 & 4.76 & .28 & .56 & 4.28 & .20 & .45 & 5.62 \\
\hline 4 & .26 & .51 & 4.83 & .23 & .57 & 5.08 & .17 & .38 & 5.76 \\
\hline 5 & .25 & .60 & 5.56 & .20 & .46 & 5.72 & .94 & .33 & 6.66 \\
\hline 6 & .23 & .61 & 5.85 & .14 & .49 & 6.25 & .12 & .28 & 7.89 \\
\hline 7 & .19 & .45 & 7.55 & .22 & 45 & 7.03 & 12 & .33 & 8.09 \\
\hline 8 & .20 & .49 & 8.24 & .19 & .49 & 8.08 & .10 & .31 & 7.69 \\
\hline 9 & .20 & .49 & 8.66 & .15 & .43 & 8.60 & .12 & .34 & 8.40 \\
\hline 10 & .22 & .54 & 9.79 & .15 & .44 & 9.32 & .08 & .26 & 8.55 \\
\hline 11 & .24 & .50 & 10.48 & .15 & .49 & 9.97 & .13 & .35 & 9.63 \\
\hline 12 & .20 & .56 & 11.24 & .19 & .52 & 10.60 & .09 & .38 & 9.76 \\
\hline 13 & .26 & .60 & 11.11 & .26 & .65 & 11.49 & .17 & .42 & 10.10 \\
\hline 14 & .42 & .52 & 11.56 & 49 & .72 & 12.65 & 22 & 41 & 10.76 \\
\hline
\end{tabular}

Downloaded from the Digital Conservancy at the University of Minnesota, http://purl.umn.edu/93227. May be reproduced with no cost by students and faculty for academic use. Non-academic reproduction requires payment of royalties through the Copyright Clearance Center, http://www.copyright.com/ 
The biggest difference between the solutions from the different sessions is that Pig moves from its position in Session 1 to be near Cat and Dog.

The rank values assigned to Pig were investigated to determine why this point moved. Since the data were row-conditional, a squared correlation between distances and disparities is computed by ALSCAL for each target. The $R^{2}$ value for $P i g$ was the lowest or all animals in both sessions $\left(R^{2}\right.$ for Session 1 Pig $=.759$, mean of others $=.888$; $R^{2}$ for Session 2 Pig $=.865$, mean of others $=$ .913). The difference was smaller in Session 2 , indicating that $P$ ig was perceived more consistently during Session 2.

Consistency of stimulus perception was examined by computing Kendall's Tau correlations between the ranks assigned to an item in the two sessions. Separate coefficients were computed for an item both as a stimulus ranked in relation to other targets and as a target. In each case, the tau coefficient for Pig was smaller than the mean of the others (for targets $\tau_{\mathrm{pig}}=.52$, $\tau_{\text {others }}=.69$; for stimuli $\tau_{\mathrm{Pig}}=.54, \tau_{\text {others }}=.63$ ), although some stimuli did have smaller values. These statistics indicate that Pig was unstable in the configuration

Figngre 1

Selected Animals Set, Session 1 Configuration

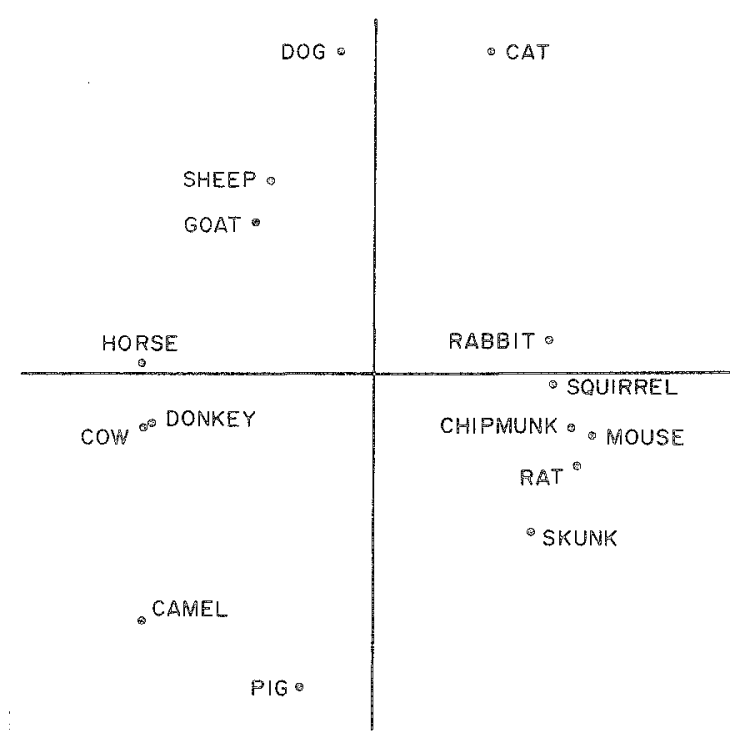

Fignare 2

Random Animals Set, Session 1 Configuration

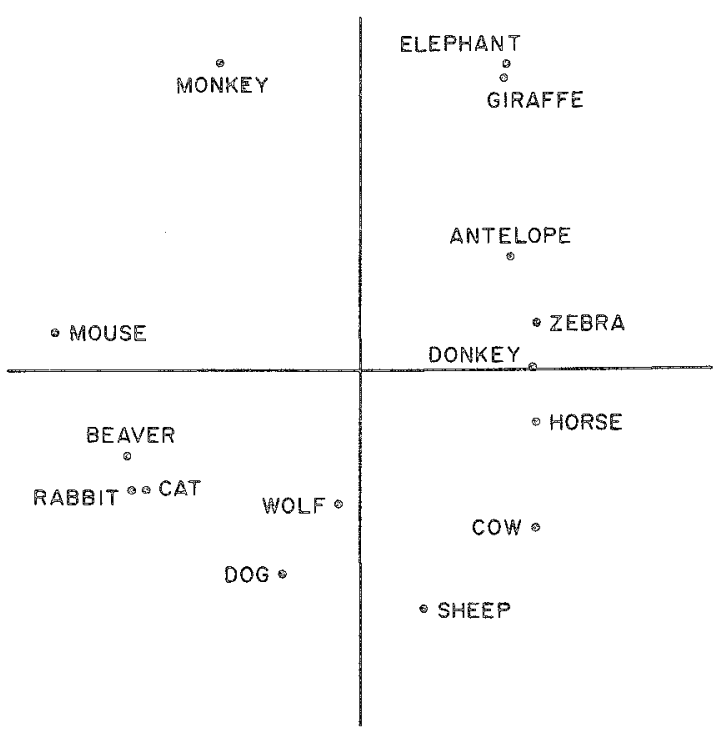

because it was rated with more variability than were the other stimuli.

Figure 2 presents the solution obtained from the complete ordering of the RA set in Session 1 (stress for Session $1=.205$, stress for Session $2=.161$ ). Figure 3 presents the solution obtained from the complete ordering of the RN set in Session 1 (stress for Session $1=.265$, stress for Session $2=.197$ ). There are no clear differences between the solutions in either of these sets. The stress values for the different sets are similar, although the value for the RN set is quite a bit higher in Session 1 (probably due to unfamiliarity with this set of stimuli). This difference decreases in Session 2, as familiarity increases. These solutions are all fairly good. The points cluster, and the clusters are sensible and predictable.

\section{Conniparing Cong}

In order to compare the configurations from the two sessions, distances between points were computed and compared, as they are invariant to rotation. The mean absolute difference (MAD) between distances was used to compare configurations. Criterion MAD values between Sessions 1 and 2 
Figure 3

Random Nouns Set, Session 1 Configuration

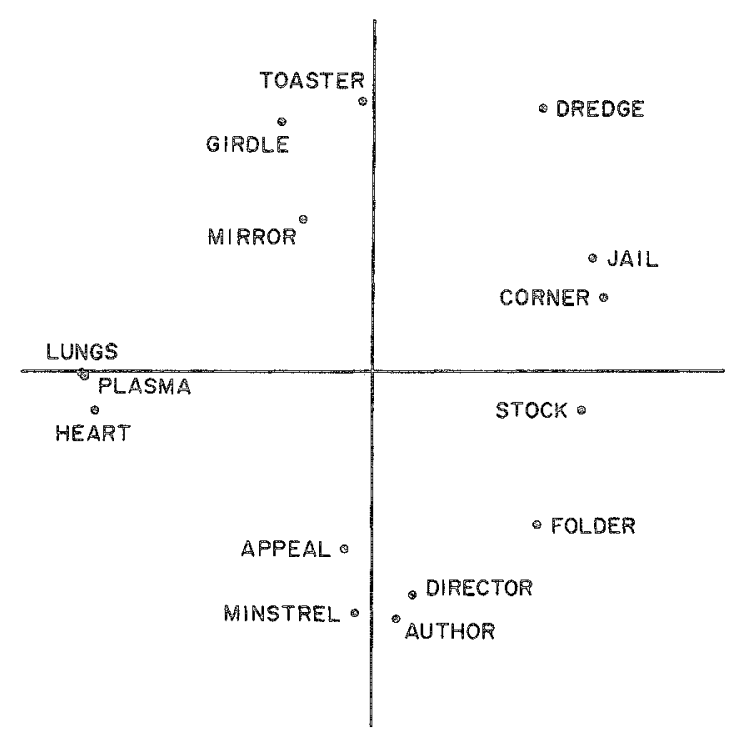

were computed $\left(\mathrm{MAD}_{\mathrm{SA}}=.54, \mathrm{MAD}_{\mathrm{RA}}=.40\right.$, $M A D_{R N}=.41$ ). The difference between the RA and RN set and the SA set is partially due to the deviant point Pig. After it was deleted, the SA MAD value dropped to .472 .

\section{Models of Judgmental Limitation}

Limitations to perception and judgment may assume several forms. The following perceptual model was used to reflect this. The judgments made about a given subset are determined by a subject's internal order; the ranks assigned to these stimuli concur with the internal order. The rest of the stimuli are not ordered in the reference to the target and are assumed to be tied. The task forces the ties to be broken, which is done in an arbitrary or random fashion, and this introduces noise into the data. Solutions should not be affected by retieing the ranks that were united in a random fashion. The ties imposed on the data were not broken in the analysis (the secondary approach of Kruskal, 1964). This approach was used because it most accurately represents the workings of the hypothesized restrictions.
There were four rules used to restrict the data.

Type 1: Ranks tied above rank $R_{\mathrm{c}}$

If $R>R_{\mathrm{c}}, R=R_{\mathrm{c}}+1$

$R_{\mathrm{c}}=2,3, \ldots, 12$

Type 2: Ranks tied below rank $R_{c}$

If $R<R_{\mathrm{c}}, R=R_{\mathrm{c}}-1$;

$R_{\mathrm{c}}=3,4, \ldots, 14$

Type 3: Ranks tied between two ranks $R_{\mathrm{h}}$ and $\mathbb{R}_{1}$

If $\mathbb{R}_{1}<R<\mathbb{R}_{\mathrm{h}}, \mathbb{R}=\mathbb{R}_{1}+1$

$(l, h)=(1,14),(2,13), \ldots,(6,9) \quad[3 \rrbracket$

Type 4: Ranks above rank $R_{\mathrm{c}}$ tied to one value, ranks below or equal tied to another value

If $R>R_{\mathrm{c}}, R=R_{\mathrm{c}}+1$;

If $R \leqslant R_{\mathrm{c}}, R=R_{\mathrm{c}}$;

$R_{\mathrm{c}}=2,3, \ldots, 13$

These four rules represent proposed limitations to judgments in the CRO task. Restriction Type 1 $(\mathbb{R} T-1)$ represents a process in which only stimuli similar to the target are ordered internally. Stimuli that are not similar are not ordered but categorized only as dissimilar; rank values are randomly assigned to these stimuli. Under this restriction, dissimilar stimuli were tied to one value, while similar stimuli remained unchanged.

Restriction Type 2 (RT-2) represents a process in which only the dissimilar stimuli are ordered internally. Ranks are thus assigned in accordance with the internal order to the dissimilar stimuli, while ranks are randomly assigned to the remaining stimuli. Similar stimuli were tied together under this restriction, while the dissimilar stimuli were not altered.

Restriction Type 3 (RT-3) represents a process in which the ranks of both the very similar and the very dissimilar stimuli are defined internally, while the intermediate stimuli are not ordered. Under this restriction, intermediate values were tied together, while extreme stimuli were not changed.

Restriction Type 4 ( $R T-4)$ represents a process in which none of the stimuli have a well-defined internal order; stimuli are only defined as similar or dissimilar to the target. It is assumed that all stimuli fall into one of the two classes. Under this restriction, the stimuli were tied into a similar and a dissimilar group.

All the rules except RT-3 were used to generate 12 analyses ( $\mathrm{RT}-3$ generates six analyses, as ranks 
were altered in pairs). The configurations resulting from the partial orders (in which some stimuli are tied together) were then compared to the complete order configurations using the MAD statistic. Two additional analyses were performed, using the point (if such existed) at which subjects had less confidence in their judgments. First, ranks above this point were tied (less similar ranks tied). To determine the effect of a comparable limitation in the more similar ranks, the same number of ranks were tied in the similar end of the scale. These two analyses tested first whether the subjects could perceive where their responses became invalid, and secondly whether the location of the tied set was important.

\section{Muntidimensional Scaling Solutions:

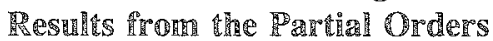

Distances from Session 2 configurations computed under the four restriction rules and the subject-defined restrictions were next compared with the distances obtained from the complete ordering configuration from Session 1 (see Table 2).

Table 2a lists the MAD values from the SA set. On the left side of the table (with the largest number of ties), the MAD values are larger than the criterion (the unrestricted MAD value) but quickly decrease. For RT-1, the MAD values begin at .59 (12 ties) but drop below the criterion (MAD = .54) with 11 values tied (.47). As the number of ties drops, the MAD value remains low. The RT2 MAD values are always higher than the RT-1 MAD values. The RT-3 MAD values lie between those from $\mathbb{R T}-1$ and $\mathrm{RT}-2$. With only a few ranks tied, these values are equal to or slightly lower than the $R T-1$ values.

RT-4 MAD values are surprisingly low. The MAD values generated under this strategy are nearly identical to those from $\mathbb{R} T-1$, between restriction values 12 and 8 . MAD values here rise quickly between 8 and 6 tied ranks; configurations with larger numbers tied of the similar ranks tied were further degraded. Although the restriction pattern is symmetric, the MAD values are not. The five values on the left side (more ranks tied in the dissimilar end) have a mean of .49 , while the five values on the right (more tied in the similar end) have a mean of .72 .

The subject-limited analyses are listed in Table 2a under "\$ $\mathbb{L}$ im." MAD value " 1 )" was obtained by tying dissimilar values above the point indicated, while value " 2 )" was obtained by tying an equal number of ranks in the similar end. The " 1 )" MAD value is as small or smaller than the lowest MAD value in the table. The MAD value with comparable restriction, "2)," is higher than most other values.

$M A D$ values from the RA set are presented in Table $2 b$. There are several differences between the two tables. The RT- 2 values are much lower here. The values under RT-2 and RT- $\mathbb{1}$ are similar, and neither rule produces consistently smaller MAD values. For RT-4 the values drop quickly to a minimum at 9 , then rise slowly. The MAD value under the subject-defined limitation " 1 )" is again small.

Table $2 \mathrm{c}$ presents results from the $\mathrm{RN}$ set. The relations between $R T-1$ and $\mathbb{R T}-2$ are similar to those in Table 2a, with RT-1 again performing better. RT-3 resembles RT-1 much more closely than it does in Table 2a. RT-4 also performs well in this set.

Next, the MAD values between the Session 2 complete order and the different Session 2 partial orders were computed. Since designs that collect data in two sessions are unusual, more generalizable conclusions are obtained by comparing restricted and unrestricted solutions from the same session. The scree test (Cartell, 1966; Kruskal \& Wish, 1978) was used to determine the point at which redundant data were added, by looking for an "elbow" in the decrease of the values.

Table 3a lists results for the SA set. For RT-1 the MAD values decrease rapidly, with a moderately clear elbow at seven tied ranks. For RT-2 there is no break in the smooth decrease in MAD values. Unlike Table $2 a$, the values for RT- 2 are uniformly smaller than comparable values for RT-1. No clear break was apparent for RT-3 or RT-4. The MAD values under the subject-defined limitation reverse the relationship found in Table 2a.

In the RA set (Table 3b) the MAD values for RT-1 show an elbow at nine ties, similar to the 
Table 2

MAD values comparing the restricted

solutions with the session 1 solutions

Table $2 a$

Selected Animals set

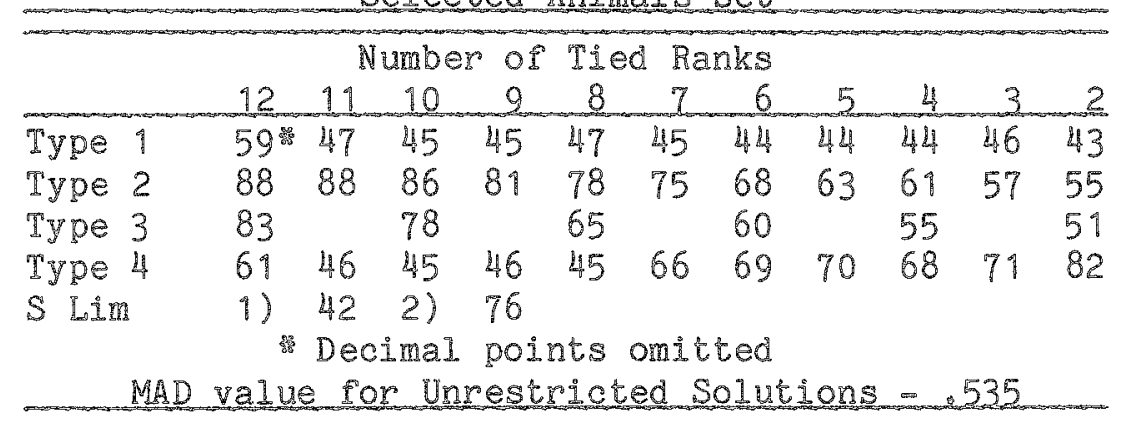

Table $2 b$

Random Animais set

\begin{tabular}{|c|c|c|c|c|c|c|c|c|c|c|c|}
\hline \multicolumn{12}{|c|}{ Number of Tied Ranks } \\
\hline & 12 & 11 & 10 & 9 & 8 & 7 & 6 & 5 & 4 & 3 & 2 \\
\hline Type 1 & 67 & 68 & 39 & 34 & 39 & 38 & 41 & 40 & 40 & 39 & 38 \\
\hline Type 2 & 65 & 67 & 59 & 53 & 51 & 40 & 34 & 36 & 36 & 36 & 37 \\
\hline Type 3 & 66 & & 58 & & 33 & & 35 & & 33 & & 35 \\
\hline Type 4 & 66 & 66 & 37 & 30 & 35 & 37 & 41 & 45 & 49 & 58 & 50 \\
\hline SLim & 1) & 37 & 2) & 44 & & & & & & & \\
\hline
\end{tabular}

Table 20

Random Nouns set

\begin{tabular}{|c|c|c|c|c|c|c|c|c|c|c|c|}
\hline \multicolumn{12}{|c|}{ Number of Tied Ranks } \\
\hline & 12 & 11 & 10 & 9 & 8 & -7 & 6 & 5 & 4 & 3 & 2 \\
\hline Type 1 & 69 & 40 & 47 & 54 & 38 & 42 & 41 & 39 & 38 & 40 & 41 \\
\hline Type 2 & 71 & 75 & 66 & 62 & 57 & 51 & 51 & 49 & 47 & 46 & 42 \\
\hline Type 3 & 60 & & 43 & & 34 & & 38 & & 49 & & 42 \\
\hline Type 4 & 40 & 37 & 48 & 65 & 47 & 45 & 45 & 55 & 68 & 78 & 76 \\
\hline S L In & 1) & 42 & 2) & 60 & & & & & & & \\
\hline
\end{tabular}

point in Table 3a. MAD values from RT-2 are similar to Table $3 \mathrm{a}$, with no perceivable break in the smooth decrease. The values for $R T-1$ and $\mathbb{R T}$ 2 are similar to one another, as in Table $2 \mathrm{~b}$. For RT-3 the decrease in MAD values is smooth, with no elbow. RT-4 has a minimal value between re- striction values 6 or 8 , when half of the stimuli are placed in each group. For the subject-defined limit, the better performance is obtained by tying ranks in the similar end of the rank order scale.

For the $R N$ set (Table 3c), the values are very similar to those in Table 3a. RT-2 again performs 
Table 3

MAD values comparing the restricted solutions with the Session 2 solution

Table $3 a$

Selected Animals set

\begin{tabular}{|c|c|c|c|c|c|c|c|c|c|c|c|}
\hline \multicolumn{12}{|c|}{ Number of Tied Ranks } \\
\hline & 12 & 11 & 10 & 9 & 8 & 7 & 6 & 5 & 4 & 3 & \\
\hline Type o & 77 范 & 64 & 61 & 60 & 58 & 48 & 49 & 39 & 37 & 34 & \\
\hline Type 2 & 62 & 58 & 53 & 48 & 43 & 36 & 30 & 20 & 16 & 07 & \\
\hline Type 3 & 66 & & 56 & & 40 & & 32 & & 28 & & \\
\hline Type 4 & 74 & 61 & 59 & 58 & 51 & 51 & 65 & 59 & 60 & 64 & \\
\hline$S \mathrm{Lim}$ & 1) & 53 & 2) & 36 & & & & & & & \\
\hline
\end{tabular}

Table 3b

Random Animals set

\begin{tabular}{|c|c|c|c|c|c|c|c|c|c|c|c|}
\hline \multicolumn{12}{|c|}{ Number of Tied Ranks } \\
\hline & 12 & 11 & 10 & 9 & 8 & 7 & 6 & 5 & 4 & 3 & \\
\hline ype 1 & 64 & 64 & 47 & 43 & 33 & 28 & 25 & 21 & 16 & 11 & 0 \\
\hline Type 2 & 60 & 63 & 52 & 44 & 3 & 36 & 31 & 20 & 10 & 05 & \\
\hline Type 3 & 61 & & 41 & & 39 & & 29 & & 20 & & \\
\hline Type 4 & 63 & 60 & 47 & 42 & 33 & 27 & 26 & 35 & 42 & 61 & 5 \\
\hline 8 LIII & 1) & 29 & 2) & 27 & & & & & & & \\
\hline
\end{tabular}

Table $3 c$

Random Nouns set

\begin{tabular}{|c|c|c|c|c|c|c|c|c|c|c|c|}
\hline \multicolumn{12}{|c|}{ Number of Tied Ranks } \\
\hline & 12 & 11 & 10 & 9 & 8 & 7 & 6 & 5 & 4 & 3 & \\
\hline Type 1 & 78 & 52 & 62 & 53 & 33 & 26 & 27 & 25 & 20 & 19 & 15 \\
\hline Type 2 & 58 & 58 & 43 & 38 & 31 & 22 & 18 & 16 & 13 & 11 & 04 \\
\hline Type 3 & 52 & & 27 & & 19 & & 17 & & 11 & & 05 \\
\hline Type 4 & 53 & 38 & 50 & 55 & 26 & 23 & 23 & 36 & 50 & 64 & 71 \\
\hline S Lim & 1) & 40 & 22 & 36 & & & & & & & \\
\hline
\end{tabular}

better than does $\mathrm{RT}-1$, although the difference is smaller. The values in RT-1 break at about 7 tied ranks. The values in RT-3 and RT-4 are similar to those in Table $3 a$. The subject-defined limit values resemble those of Table $2 \mathrm{a}^{2}$

Variance accounted for is a measure of the similarity of the recovered values to the observed data.

2Plots and statistics about solutions obtained under some of the partial orders may be obtained from the author.
$R^{2}$ is a measure of this relationship (Young \& Lewyckyj, 1979). When many values are tied, the $R^{2}$ values were low (between .61 and .70) but rose quickly; the $\mathbb{R}^{2}$ values in the restricted orders equaled or surpassed the complete order $R^{2}$ (for RT- 1 and RT-2) with seven values tied. RT-1 performed slightly better than $R T-2$. These $R^{2}$ values indicate that the recovery of the data was quite good with fully half the data tied together.

$\mathrm{RT}-1$ is the process which is supported most consistently and seems to be the most efficient rule. 
In the SA set, the MAD value with 9 values tied is equivalent to the criterion value. Under RT-2, the restriction with a comparable MAD value has 3 ranks tied. In Table $2 b$ the pattern is reversed, however. RT-2 values are lower than under RT-1. For RT-4, MAD values with a small number of similar ranks tied (and a compensatorily large number of dissimilar ranks tied) are lower than those with a large number of similar ranks tied (and a small number of dissimilar ranks tied).

The additional evidence from Table 3 supports the conclusion that RT- 1 is the most accurate model of the psychological process. When comparing RT1 and RT-2, it is clear that the MAD values break earlier under RT-1 than under RT-2. In Table 3a the break occurs with 9 values tied under RT-1 restrictions. The break for RT-2 is less obvious than for RT- $\mathbb{1}$ and occurs later, with 5 ranks tied. In the case of Table $3 b$, the break occurs with 9 or 8 values tied under RT-1 and with 7 values tied under Type 2. RT-1 configurations are more similar to the reference configuration than are the comparably restricted $\mathrm{RT}-2$ configurations.

These results stand in direct contrast to those obtained by Graef and Spence (1979). They performed a similar experiment, contrasting matrices to true configurations after deleting either small distances (corresponding to RT-2), large distances (corresponding to $\mathrm{RT}-1$ ), and medium distances (corresponding to RT-3). The important difference between their procedure and the present procedure lies in the constraining process. In their case, they eliminated the distances in question, while here the distances were constrained to be identical. As they performed their analyses in TORSCA-9 (Young, 1968), the primary approach to ties was used (Young, 1982) on such missing data. Here, the tied block of values was constrained with respect to the other values.

This deletion method was also applied to these data. Four ranks in the dissimilar end, the similar end, and the middle of the scale were deleted and the data were scaled by ALSCAL. These results are compared to some of the analyses already performed. There were only small differences between the values calculated under the two methods (Session 1 data are used for this process and compared with the Session 2 complete ordering). For the SA set the restricted low, middle, and high solutions were different for the two methods of restricting data (tied: $\mathrm{MAD}$ (low) $=.61, \mathrm{MAD}($ mid $)=.55$, MAD (hi) $=.44$; deleted: $\operatorname{MAD}$ (low) $=.64$, MAD (mid) $=.49, \operatorname{MAD}($ hi $)=.57)$. The RA set had a similar result (tied: $\mathrm{MAD}$ (low) $=.36$, MAD (mid) $=.33, \operatorname{MAD}($ hi) $=.40$; deleted: $\operatorname{MAD}($ low $)=.39, \mathrm{MAD}(\mathrm{mid})=.33, \mathrm{MAD}$ (hi) $=.40$ ). The RN set resembled the SA set (ried: $\mathrm{MAD}$ (low) $=.47, \mathrm{MAD}$ (mid) $=.41$, $\operatorname{MAD}$ (hi) $=.38$; deleted: $\operatorname{MAD}($ low) $=.87$, MAD (mid) $=.56, \operatorname{MAD}($ hi $)=.50$ ). These approaches led to similar results here, mainly differing in magnitude, with the transformation approach having smaller MAD values than the deletion approach. In each case, eliminating the low values leads to recovery which is at least as bad (usually worse) as that produced by eliminating the high values.

Graef and Spence (1979) found that the deletion of large distances (high values) was more deleterious to accurate recovery than were the low or medium distances. The differences between their study and the present one could have been produced by two factors. The first is the method of restricting data. In Graef and Spence's method, distances were eliminated from the set. This did not produce great differences in recovery when both were applied to the data here. The second source of difference lies in the nature of the data used for the analysis. In the present study, data arose from subjects with the same biases and limitations of all observed data. In Graef and Spence (1979), the data were generated by a random process, which was assumed to be consistent. Their conclusion (large distances are more important than small for the recovery of the configuration) is only valid if the same process is occurring in the small and large distances. The present study indicates that this may not be valid in real data.

\section{DỉSCRSSTIOT}

In the $S A$ and $R N$ sets, MAD values computed under RT-1 were consistently superior to RT-2MAD values; this situation was reversed in the RA set. It is difficult to state unequivically which of these models is the better. The RT-1 rule is more efficient 
in two of the three sets; this strongly supports it as the best rule.

Although RT-3 was not the most efficient rule, it was generally close to being so. The solutions obtained under this rule were better than those obtained using RT-2 when there were a large number of restrictions imposed upon the data, but the MAD values obtained here were never better than those obtained under RT-1. If more dissimilar than similar ranks were tied, MAD values under this rule might be higher.

The efficiency of $R T-4$ is the most interesting result of the investigation. If equal size categories were used and the data were sorted into two equal size groups, the MAD values were quite reasonable. In some cases they were better than the MAD values obtained under RT-1 or RT-2. The peculiar drop in MAD values under this rule found in Tables $2 a$ and $3 a$ is confusing but probably can be eliminated by forcing the similar set to be smaller than the dissimilar.

The subjects were able to accurately determine where their ratings became unstable. In most cases, tying ranks above the subject-defined points resulted in a MAD value that was among the lowest overall and much better than that obtained by tying an equal number of ranks in the high similar end of the rank ordering. This indicates that the subjects are good judges of information accuracy and reliability.

There is some evidence, primarily found in Table 1, that the most dissimilar rank has some special status. This is not inconsistent with the general theme of this discussion but merely indicates that subjects are quite sensitive to the most different stimuli.

No model is supported conclusively by the evidence from all three sets. However, it is clear that the configuration may be adequately recovered from data which are quite degraded. When many stimuli are tied to one common value, the test MAD values still are as high as is the criterion MAD value. Recovery is never perfect but often is extremely good.

The comparison made between the values generated under a deletion approach (like that of Graef
\& Spence, 1979) and a transformation approach (as used here) reveals little difference between the two methods. The rank orders of the small, medium, and large distance changes are usually similar, indicating that the same process is occurring in both methods. The different results obtained by Graef and Spence (1979) must then be due to a difference in process. The remaining difference between their study and the present discussion involves the data, which are from distinct processes. The assumption of consistent process made by Graef and Spence (1979) is not warranted. Subjects are not performing the same task in all portions of the rank order task, and thus conclusions drawn from artificial data may be generalized only with great care.

\section{Conderisiones}

The results of the investigation suggest certain methods which may be used to reduce the information load that subjects are under in performing the typical MDS task. The number of judgments can be reduced in several ways. Subjects could be asked to order the five or six stimuli which they considered the most similar to the target. The present work suggests that the solution which would be obtained in this circumstance would be similar in many important ways to the configuration obtained in the full ranking case. The evidence from the solutions in which only the ranks that the subjects considered reliable were used (the others being assumed equal and most dissimilar) suggests that solutions obtained under this procedure would be quite satisfactory. A second suggestion consists of first asking subjects to select the dissimilar stimuli and to rank them. The similar stimuli would then be ranked. The intermediate stimuli would be ignored in the ranking procedure and would be treated as tied ranks during the scaling. The information load in this task would be considerably reduced compared to the normal MDS task and has characteristics similar to those of RT-3. This suggestion has support from the experimental results. In either case, subjects would be fresher and more alert for longer periods. This is probably worth the insub- 
stantial loss in precision of the final configuration, which could be offset in part by using more subjects and thus obtaining more data points. Additionally, more target stimuli could be scaled by the subjects. Since each subject is probably only effective and accurate in making a limited number of judgments, these judgments most profitably consist of scaling a few stimuli in reference to a large number of targets.

A second suggestion for reduction in cognitive overload is derived from RT-4. This entails having the subjects sort the stimuli into wo piles in relation to each target, one pile being similar stimuli and the other dissimilar to the target. The piles should be constrained to be of either equal size or of a size so that the "more similar" pile is slightly smaller than the "Iess similar" pile. A simpler and more straightforward method of gathering data is difficult to imagine. The subject must make a simple binary decision for each stimulus, which is unlikely to tax the attention span or concentration of subjects; it is also very quick. Again, any subsequent loss in precision of recovery of infomation can be offset by the acquisition of more subjects.

\section{Preferes}

Cattell, R. B. The scree test of the number of factors. Multivariate Behavioral Research, 1966, 1, 245-276.

Graef, J., \& Spence, I. Using distance information in the design of large multidimensional scaling experiments. Psychological Bulletin, 1979, 86, 60-66.

Green, P.E., \& Rao, V.R. Applied multidimensional scaling. New York: Holt, 1972.

Henley, N. M. A psychological study of the semantics of animal terms. Journal of Verbal Learning and Verbal Behavior. 1969, 8, 176-184.

Kruskal, J. B. Multidimensional scaling by optimizing goodness of fit to a nonmetric hypothesis. Psychometrika, 1964, 29, 1-28.
Kruskal, J. B., \& Wish, M. Multidimensional scaling (Sage University Paper Series on Quantitative Applications in the Social Sciences, 07-011). Beverly Hills CA: Sage Publications, 1978.

Shepard, R. N., Kilpatric, D. W., \& Cunningham, J. P. The internal representation of numbers. Cognitive Psychology, 1975, 7, 82-138.

Spence, I., \& Domoney, D. W. Incomplete designs for nonmetric multidimensional scaling. Psychometrika, $1974,39,469-490$.

Takane, Y. M., Young, F. W., \& deleeuw, J. A. A nonmetric individual differences algorithm with optimal scaling features. Psychometrika, 1977, 42, 767.

Torgerson, W. S. Theory and methods of scaling. New York: Wiley, 1958.

Young, F. W. A FORTRAN IV program for nonmetric multidimensional scaling (L. L. Thurstone Psychometric Laboratory Report No. 56). Chapel Hill NC: University of North Carolina, L. L. Thurstone Psychometric Laboratory, 1968.

Young, $\mathbb{F}$. W. Scaling replicated conditional rank-order data. Sociological Methodology, 1975, 6, 129-170.

Young, F. W. Personal communication, 1982.

Young, F. W., \& Lewyckyj, R. ALSCAL-4User's Guide. Carrboro NC: Data Analysis and Theory Associates, 1979.

\section{Ach}

This paper is adapted from a paper presented at the 1980 meeting of the P sychometric Society, Lowa City IA, May 26-28, 1980. Cynthia Null, Forrest Young, Joe Lee Rodgers, and two reviewers made suggestions which greatly improved both the substantive ideas and the presentation.

\section{Adurhor's AddreSS}

Send requests for reprints or further information to Paul Thompson, L. L. Thurstone Psychometric Laboratory, Davie Hall 013-A, University of North Carolina, Chapel Hill NC 27514. 